\title{
Service based Model using Context Awareness for Ubiquitous Computing
}

\author{
Waquas Saeed \\ TPS Pakistan Pvt. Ltd. \\ Karachi, Pakistan
}

\author{
Rameez Hussain \\ TPS Pakistan Pvt. Ltd. \\ Karachi, Pakistan
}

\begin{abstract}
A semantic network formed relationships and structures for ubiquitous computing which provide mobile services from one node to another. Mobile agents - a kind of ubiquitous computing, entitle service driven architecture with context awareness regardless of the physical interaction and location of users. Based on conceptual service model, context awareness semantic can easily be developed for service description, publishing and discovery for mobile agents discussed in this paper. Context awareness semantics using service based model is a function for every mobile agent to invoke and a necessary foundation of the ability of service adaption for computing everywhere.
\end{abstract}

\section{Keywords}

Requirements, context-awareness semantic, mobile agents, ubiquitous computing, service adaption

\section{INTRODUCTION}

Computing at present becoming pervasive and ubiquitous environments in which devices, software agents and services all are expected to seamlessly integrate and cooperate in support of human efforts, and providing a platform with automatic service discovery regardless of user's context awareness [1]. Therefore, more advanced services can be provided when they interact, but service adaption can only be achieve when devices have user's behavior and preferences.

In ubiquitous computing, the physical context is very important which acquired using sensors data, data gathered in collaboration with other agents and through semantics network. Besides that, the role of context has recently gained great importance in the field of ubiquitous computing. Context in any information can be an object, an entity or a data which when interact and collaborate, can lead to new piece of information surrounded by user's perceptive and needs. A lot of research work has been done to make applications context aware so that they can adopt and learn to different situations and be more accessible to user's needs [4].

In general, agents are applications, services and devices which interact and achieve a user task. Ubiquitous environments are characterized with different context which include physical, environmental, informational, system, applications and social types [4]. A service in this environment should be able to acquire information and reason about these contexts where by acquiring new services, agent should adapt behavior and learn to react.

In this paper, we propose a conceptual service based model for ubiquitous computing. In our conceptual model, Service Transformation (ST) - a function based service which works in two modes. Active Service Transformation (STav) service is used when agents are interacting and collaborating with each other to share information, where as Passive Service
Transformation (STpv) service is used to gather new source of data and discover information and formed into a new service inactively. Service Transformation (ST) is a layer which interacts with different nodes and maintain internal database for new services, providing a new agent a service to ubiquitous environment.

\section{CONCEPTUAL MODEL AS SERVICE}

Service mobility in ubiquitous environment is one of the problems which require mobility of user's without the physical aspect, and to provide different services system should be capable of self-adaption. There are several context aware devices which interact with each other in order to share information primarily based on agent based mechanism. On the other hand, service as an agent is a new paradigm which provides simplified way to compose new services. They not only provide new information to users but can also able to formed new services. In this way, user's perspective can easily be gathered when a new request is found and registered as a service token for other devices. The below section describes the architecture and working of conceptual model.

\section{ARCHITECTURE}

We used service as a concept which comprises of three components as given in Figure 1:

\section{Service Transformation (ST)}

A main component layer responsible of managing services, interaction and collaboration. It also enables services to formed new services as agent.

\section{Active Service Transformation (STav)}

A low level component layer which interacts and collaborate information with different nodes.

\section{Passive Service Transformation (STpv)}

A low level component layer which passively work with and formed new services as an agent. It also gathers new information and data.

Service Transformation (ST) is a service which mainly has two elements:

\section{Agent Registration}

When a new service is formed, necessary data is provided to register and publish service as an agent.

\section{Service Data}

Low level layers have general description. It includes profile identity, profile data, profile service function.

\section{WORKING MODEL}

This section describes the overall working of proposed conceptual model as service. 


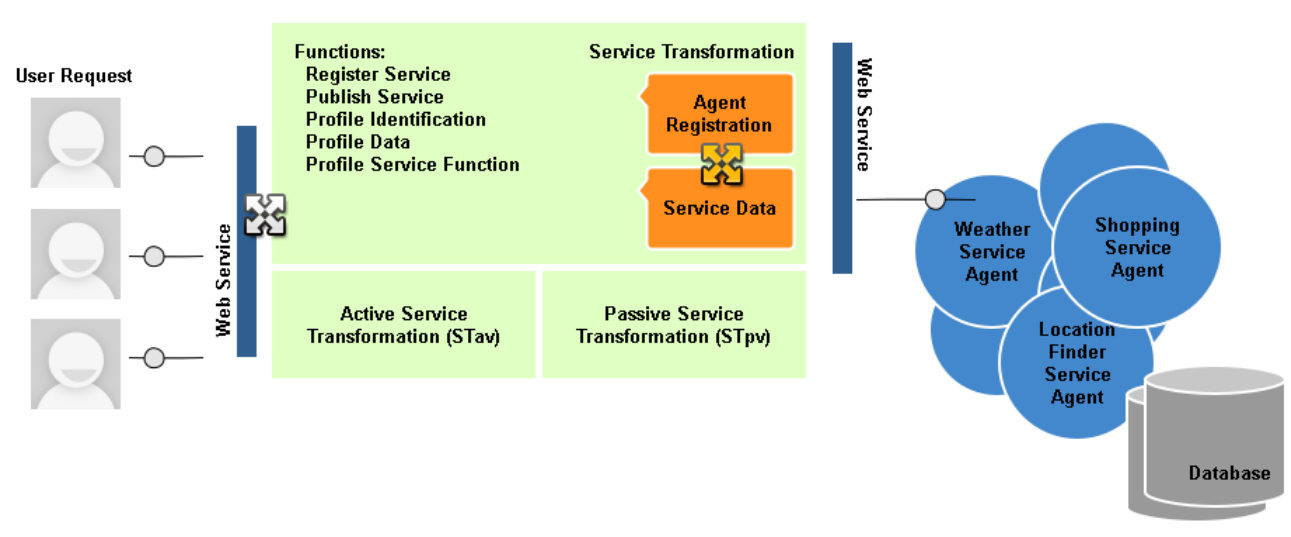

Figure 1: Architecture of Service based Model

When a context aware devices or agents initiate a request, it is transformed into well defined format which interacts with several intermediate nodes to achieve user's task. The Service Transformation (ST) is a service which constantly querying in a passive mode to formed new service. If, any initiated request is not found in internal database, Active Service Transformation (STav) enable its self-adaption service and collaborate with external information servers or web services. There are two benefits of Service Transformation (ST):

- When a hit is made to external informational servers, necessary information is transformed by Service Transformation (ST) layer regardless of protocol and specification.

- The same information is then composed into well defined structure for Agent Registration and Service Data. This metadata is generated on runtime without any processing.

When a user's task is completed, a call is made to Service Transformation (ST) which invokes a service call to Passive Service Transformation (STpv) to form a new service as agent. Once the service is formed, service as an agent is registered and published its necessary information to external servers which may used for collaboration and providing new service. This conceptual model is a simplified model based on services which do not require any special structure to locate and provide service. The internal database is a central location which provides information about services, their function and specification in a simplified manner. The same model can be made more flexible if another service model is constructed which focuses on geographical location, and provide more interacting service in different languages.

\section{CONCLUSION AND FUTURE WORK}

In this paper, service as an agent model is proposed which provide easy and simplified implementation across the different nodes. In this way, user's task is achieved with collaboration and interaction while composing new service as an agent. The Service Transformation (ST) is a mechanism used to formalized new set of function as service.

The future work will include more flexible services based on language and geography where multi source paradigm is used to compose new services. This will open a new paradigm for language filtration based on different user's request while achieving user's goal in simplified manner.

\section{REFERENCES}

[1] Weidong Wen, Yanxiange He, Li Li. A mobile service language based on mobile agent for ubiquitous computing. 2008 International Conference on Computer Science and Software Engineering, pp. 1186-1188.

[2] Shenghong Yang, Jia Yan. Ontology Component Development. 2009 International Conference on Computational Intelligence and Natural Computing, pp. 92-95.

[3] Wei Wei, Saiyu Qi, Yong Qi, Wei Wang, Min Xi. Allotropy Programming Paradigm for Ubiquitous Computing Environment. 2007 International Conference on Convergence Information Technology, pp. 514-521.

[4] Anand Ranganathan, Roy H. Campbell. A Middleware for Context-Aware Agent in Ubiquitous Computing Environments.

[5] Jerome Euzenat, Jerome Pierson, Fano Ramparany. A Context Information Manager for Pervasive Computing Environment.

[6] Harry Chen, Tim Finin, Anupam Joshi. The Role of the Semantic Web in Pervasive Context-Aware Systems. 